Revue de droit comparé du travail et de la sécurité sociale

$3 \mid 2020$

La Directive 2019/1158 du 20 juin 2019 concernant l'équilibre entre vie personnelle et vie privée des parents et des aidants

\title{
La consécration constitutionnelle des principes $d u$ système de retraite
}

\section{Anna Aleksandrova}

\section{OpenEdition}

Journals

Édition électronique

URL : https://journals.openedition.org/rdctss/1036

DOI : $10.4000 /$ rdctss. 1036

ISSN : 2262-9815

Éditeur

Centre de droit comparé du travail et de la sécurité sociale

Édition imprimée

Date de publication : 1 novembre 2020

Pagination : 200-205

ISSN : 2117-4350

Référence électronique

Anna Aleksandrova, "La consécration constitutionnelle des principes du système de retraite ", Revue de droit comparé du travail et de la sécurité sociale [En ligne], 3 | 2020, mis en ligne le 01 novembre 2021, consulté le 11 novembre 2021. URL : http://journals.openedition.org/rdctss/1036 ; DOI : https:// doi.org/10.4000/rdctss. 1036

\section{(c) (i) $\odot$}

Revue de droit comparé du travail et de la sécurité sociale est mise à disposition selon les termes de la Licence Creative Commons Attribution - Pas d'Utilisation Commerciale - Pas de Modification 4.0 International. 


\section{ANNA ALEKSANDROVA}

UNIVERSITÉ d'ÉTAT DE PENZA

\section{LA CONSÉCRATION CONSTITUTIONNELLE DES PRINCIPES DU SYSTÈME DE RETRAITE ${ }^{1}$}

Au cours du premier semestre 2020, les pouvoirs publics en Russie, comme dans la plupart des pays, ont accordé une attention particulière à la lutte contre le COVID-19. Un nombre significatif de règlements concernant la protection sociale ont ainsi été adoptés.

Dans le même temps, des modifications ont été apportées à la Constitution de la Russie, y compris l'instauration des principes généraux du système de retraite. Ces changements sont relativement importants et, à long terme, vont probablement influencer le développement de la législation russe sur les retraites si la stabilité constitutionnelle perdure.

Dans cet article, nous examinerons les caractéristiques du droit russe de la sécurité sociale, notamment l'absence d'un Code et la consécration normative des principes de base (I), avant d'analyser les normes constitutionnelles régissant actuellement les relations de sécurité sociale en général, et de la retraite en particulier (II).

\section{L'ABSENCE DE CODIFICATION DU DROIT DE LA SÉCURITÉ SOCIALE EN RUSSIE}

Il n'y a jamais eu en Russie de Code de la sécurité sociale. Cette lacune importante complique l'application de la loi dans la mesure où il existe de nombreuses règlementations de même niveau (par exemple les lois fédérales) qui régissent parfois différemment les mêmes relations.

L'existence d'un Code contenant les principes de base du droit de la sécurité sociale permettrait sans doute de mieux résoudre les conflits qui surgissent. II n'est toutefois pas ici question d'aborder la complexité de la législation sociale et son inaccessibilité à la compréhension des personnes - bénéficiaires des pensions et des prestations.

L'absence de Code de la sécurité sociale est plutôt une exception qu'une règle caractéristique du système juridique russe. La plupart des branches du droit russe sont en effet codifiées (droit du travail, droit civil, droit criminel, droit familial, etc.), ce qui rapproche le droit russe du système juridique continental. Rappelons que les premiers Codes ont été introduits en Russie il y a plus de 100 ans.

1 Cette étude a été financée par RFBR, numéro de projet 20-011-00252. 
En matière par exemple de droit du travail (le plus approchant du droit de la sécurité sociale dans son contenu), le premier "Code des lois de travail " a été adopté en 1918. Actuellement, le Code du travail de la Fédération de Russie de $2001^{2}$ est en vigueur, et sa section I ("Dispositions générales ») consacre les buts et objectifs de la législation du travail (art. 1), ainsi que les principes fondamentaux de la réglementation juridique des relations de travail (art. 2).

Le droit de la sécurité sociale a été élaboré en Russie dans la seconde moitié du $X X^{e}$ siècle. Jusqu'au milieu des années 1950, les relations dans le domaine de la sécurité sociale étaient principalement régies par des règlements : décrets du gouvernement soviétique, réglementations syndicales (car les syndicats soviétiques étaient chargés de la gestion de l'assurance sociale en 1933), etc. Les seules exceptions étaient les dispositions de la Constitution de I'URSS de 1936, qui consacraient le droit des citoyens à un soutien financier en cas de vieillesse, d'invalidité, de perte de soutien familial (art. 120).

En 1956, la loi de l'URSS sur les pensions d'État a été adoptée, elle énonçait de manière systématique les dispositions relatives aux pensions, les types et motifs d'affectation. En 1990, à l'ère des réformes économiques et politiques, la loi sur les pensions d'État ${ }^{3}$ a à son tour été adoptée en Russie. Elle a consolidé des innovations importantes, par exemple le principe de l'indexation des pensions, la création d'un nouveau type de pension sociale susceptible d'être versée aux personnes sans expérience professionnelle, etc.

Puis, le développement de la législation s'est traduit par une augmentation du nombre de lois fédérales régissant les prestations de retraite. En 1993, une loi a ainsi été adoptée, établissant des règles spéciales pour les pensions du personnel militaire ${ }^{4}$. Lors de la réforme des retraites de 2001, un autre ensemble de lois a été adopté pour remplacer la loi de 1990. Les pensions financées par les caisses de l'assurance sociale étaient régies par la loi fédérale $n^{\circ} 173^{5}$, et les pensions financées par le budget de l'État par la loi fédérale $\mathrm{n}^{\circ} 166^{6}$.

Dans les années suivantes, il y a eu une augmentation aléatoire des lois sur les retraites. Ainsi, aujourd'hui, à la place de la loi n ${ }^{\circ} 73$, deux lois sont en vigueur : celle du 28 décembre 2013 surlespensions d'assurance ${ }^{7}$ etcellesurlespensionscapitalisées du 28 décembre $2013^{8}$ (sans omettre les nombreuses lois régissant toutes sortes de

2 Code du travail de la Fédération de Russie : http://www.consultant.ru/document/cons doc LAW 34683/

3 Loi de la Fédération de Russie du 20 novembre 1990 n³40-1 sur les pensions d'État : http://www.consultant.ru/document/cons doc LAW 28/

4 Loi de la Fédération de Russie du 12 février 1993 n4468-I.

5 Loi fédérale du 17 décembre $2001 n^{\circ} 173$ sur les pensions de travail : http://www.consultant. ru/document/cons doc LAW 34443/

6 Loi fédérale du 15 décembre 2001 n¹66 sur la prestation de pension de l'Etat: http://www. consultant.ru/document/cons doc LAW 34419/

7 Loi fédérale du 28décembree $2013 n^{\circ} 400$ sur les pensions d'assurance : http://www. consultant.ru/ document/cons doc LAW 156525/

8 Loi fédérale du 28 décembre $2013 n^{\circ} 424$ sur la pension capitalisée : http://www.consultant. ru/document/cons doc LAW $156541 /$ 
procédures en vue de l'obtention des pensions). Tout ceci a permis aux experts de conclure à «la décodification » de la législation sur la sécurité sociale en Russie?.

L'idée de codifier la législation russe en matière de sécurité sociale s'est régulièrement manifestée ces dernières années ${ }^{10}$. Des propositions ont été avancées concernant la codification de certains grands ensembles réglementaires, par exemple la législation de retraite, la législation sur les allocations familiales, etc. Mais, à ce jour, aucune tentative n'a été entreprise par le Parlement fédéral dans ce sens.

Dès lors, la consolidation des principes du système de retraite au niveau constitutionnel acquiert une importance particulière. En l'absence de Code, les normes constitutionnelles peuvent devenir des lignes directrices qui seront le vecteur du développement ultérieur de la législation sur les retraites, et qui seront utilisées par la jurisprudence dans le règlement des différends concernant la retraite.

\section{LA RÉGLEMENTATION CONSTITUTIONNELLE DES RETRAITES EN RUSSIE}

LaConstitution delaFédération deRussie, adoptéele12décembre1993,a proclamé la Russie «Étatsocial » dont la politique «vise à créer les conditions qui garantissentune vie digne et un développement humain libre " (partie 1 de l'art. 7) ${ }^{11}$. Dans la partie 2 de l'article 7, cette disposition est concrétisée par l'énumération des mesures prises par l'Etat ; il s'agit particulièrement de l'établissement de pensions d'État. Cet article fait partie du chapitre 1 de la Constitution qui établit les bases de l'ordre constitutionnel du pays.

Dans le deuxième chapitre consacré aux droits et libertés de l'individu, le droit à la sécurité sociale est prévu par l'art. 39 (partie 1), selon lequel « chacun a droit à la sécurité sociale en cas de vieillesse, maladie, invalidité, perte d'un soutien de famille, pour élever des enfants et dans d'autres cas prévus par la loi ». L'utilisation du terme "chacun » indique que le droit à la sécurité sociale est reconnu à tout individu, indépendamment de la nationalité, du sexe, du statut social, etc.

Les règles des articles 7 et 39 ont été incluses dans la version initiale de la Constitution de la Russie de 1993 et sont restées inchangées jusqu'à présent puisqu'elles figurent aux chapitres 1 et 2 de la Constitution. Pour ces chapitres (ainsi que pour le chapitre 9), il existe une procédure spéciale de modification d'une complexité exceptionnelle. Pour les chapitres de 3 à 8 , la procédure est plus simple, bien qu'elle prévoie l'adoption d'une loi constitutionnelle fédérale et l'approbation d'amendements par les parlements régionaux.

9 E.G. Toutchkova, Le droit de la sécurité sociale de la Russie, Moscou, 2016, p. 88.

10 " Réformes des retraites en Europe centrale et orientale et rôle de l'Organisation internationale du Travail (Remarques liminaires de I'OIT) », in E.G. Tuchkova (dir.), Normes internationales et russes en matière de pensions: Analyse comparative, Moscou, 2013, p. 23.

11 Constitution de la Fédération de Russie du 12 décembre 1993 : http://www.consultant.ru/ document/con s doc LAW 28399/ 
Lorsqu'en janvier 2020 le Président de la Russie a avancé l'idée de modifier la Constitution, il s'agissait précisément de modifier les chapitres de 3 à 8 . Les normes contenant les principes du système de retraite en Russie ont été incluses à l'article 75 de la Constitution (qui, dans sa version originale, était consacré aux questions financières et fiscales).

Dans sa version finale, l'article 75 a ainsi été complété par la partie 5 (établissant des garanties du salaire minimum), la partie 6 (sur les principes du système de retraite) et la partie 7 (sur les garanties d'assurance sociale obligatoire, l'aide sociale ciblée et l'indexation des prestations sociales).

Bien entendu, il serait plus cohérent du point de vue de la procédure juridique de placer les principes du système de retraite dans les articles 7 sur l'État social ou 39 sur le droit à la sécurité sociale. Mais, ce sont des facteurs conjoncturels qui ont déterminé le processus de réforme, notamment la volonté des autorités d'apporter rapidement des modifications au texte de la Constitution.

En effet, pour modifier les chapitres 1, 2 et 9 de la Constitution, une institution spéciale doit être convoquée - l'Assemblée constitutionnelle - qui doit élaborer un projet de nouvelle Constitution et l'adopter, ou le soumettre à un référendum (conformément à la partie 2 de l'article 135 de la Constitution de 1993). La procédure de convocation de l'Assemblée constitutionnelle doit être déterminée par la loi constitutionnelle fédérale, mais une telle loi n'a pas encore été adoptée (bien que la Constitution soit en vigueur depuis 27 ans).

Ainsi, pour apporter rapidement des modifications à la Constitution, les autorités ont décidé de ne les introduire que dans les chapitres de 3 à 8 . Il faut préciser que le nombre d'amendements est relativement important, et que ces derniers concernent non seulement la sphère sociale, mais aussi la sphère politique, les questions d'organisation des pouvoirs publics, leurs compétences, etc.

La procédure même d'introduction des amendements a suscité de nombreuses critiques. En particulier, la Constitution ne prévoit pas de référendum pour modifier les chapitres 3 à 8.

De plus, alors que le pays était en situation de pandémie, le référendum s'est déroulé pendant plusieurs jours (au lieu d'une journée); des bureaux de vote mobiles et le vote par Internet ont été organisés, etc. Nonobstant ces différentes observations, la loi portant modification de la Constitution a été adoptée le $1^{\text {er juillet }}$ 2020 et est effectivement entrée en vigueur.

Les principes d'organisation du système de retraite, inclus dans la partie 6 de l'art. 75 de la Constitution de la Fédération de Russie, sont les suivants:

Universalité : chacun a droit à une pension. Les personnes qui participent au régime d'assurance sociale peuvent recevoir une pension s'ils ont atteint l'âge de retraite, ont cotisé pendant une période établie et ont cumulé des points de retraite (calculés en fonction du montant des cotisations). Les militaires et les fonctionnaires peuvent recevoir une pension de l'Etat s'ils ont l'ancienneté requise et pour les autres personnes dans l'incapacité de travailler ou n'ayant pas acquis les droits nécessaires, elles bénéficient d'une pension relevant de l'assistance. 
Équité : en matière de retraite, elle peut être considérée comme la corrélation entre le montant de la pension et celui de la «contribution» d'un individu. Jusqu'en 2020, la Constitution de la Russie n'évoquait nullement ce principe, dont la consécration doit être saluée.

Solidarité entre générations : formulé aux $X I X-X X^{e}$ siècles, ce principe signifie que les individus qui travaillent actuellement soutiennent les retraités en payant les cotisations d'assurance. Ainsi, les générations futures devront soutenir ceux qui travaillent aujourd'hui. La consolidation de ce principe au niveau constitutionnel revêt une importance particulière car, il a été remis en question ces dernières années, et des projets de révision du "contrat de générations » ont été lancés. En Russie, au début des années 2000, comme dans la plupart des pays d'Europe de l'Est, le gouvernement a tenté d'introduire un élément de capitalisation obligatoire dans la structure des pensions, réduisant ainsi le rôle du principe distributif basé sur le principe de solidarité entre générations.

En Russie, depuis 2002, 6\% des primes d'assurance (sur 22\% versées par l'employeur à la Caisse de retraite) ont été destinées à la formation de la partie capitalisée des pensions. Cela a provoqué un déficit de la Caisse de retraite et une pénurie de fonds pour financer les paiements aux retraités actuels. En 2010, de nombreux pays d'Europe de l'Est ont décidé de réduire la partie capitalisée obligatoire des pensions, voire même de la supprimer. En 2013, la législation a prévu pour le travailleur le droit de choisir entre continuer à verser des cotisations pour la pension capitalisée ou le refuser.

Depuis 2014, on constate un «gel » de l'épargne retraite, ce qui signifie que même pour les personnes qui souhaitent constituer une pension capitalisée, $6 \%$ de ces cotisations ne sont pas transférées et iront à la formation d'une pension d'assurance.

Ainsi, selon les experts de I'OIT, « les erreurs commises pendant la planification et la mise en œuvre de ces réformes ont entraîné des coûts de transition importants qui, face aux déficits budgétaires, ont contraint un certain nombre de pays à revoir ces réformes et à revenir au développement prioritaire de l'assurance publique ${{ }^{12}}^{12}$. Les modifications apportées à la législation russe, y compris les amendements à la Constitution, confirment cette conclusion.

Pour ce qui concerne la Constitution de la Russie, son article 75 (partie 6) prévoit désormais l'indexation obligatoire des pensions (annuellement). Le gouvernement ne pourra plus annuler l'indexation ou la remplacer par un paiement forfaitaire (comme il l'a fait en 2016). L'indexation obligatoire est également prévue pour les prestations sociales (partie 7 de l'art. 75 de la Constitution).

La Constitution a été en outre complétée par l'article 75.1 qui rassemblent les caractéristiques de l'État social, telles que le partenariat social, la solidarité économique, politique et sociale, etc.

12 « Réformes des retraites en Europe centrale et orientale et rôle de l'Organisation internationale du Travail », in E. G. Tuchkova (dir.), Normes internationales et russes en matière de pensions: Analyse comparative, op. cit., p. 3. 


\section{FÉdÉRATION DE RUSSIE}

Bien entendu, il serait plus logique de placer ces dispositions dans l'art. 7 de la Constitution. Mais, dans tous les cas, leur consolidation au niveau constitutionnel doit être appréciée positivement.

Il faut toutefois s'interroger sur le fait que, si aujourd'hui les autorités peuvent modifier si facilement le texte constitutionnel en complétant les dispositions sur les fondements de l'ordre constitutionnel et des droits de l'individu par de nouvelles normes, leurs successeurs pourraient tout aussi aisément changer ces principes de base aujourd'hui loués... 